Relations industrielles

Industrial Relations

\title{
Le mouvement de restructuration du travail, par Paul Deschênes, Québec, ministère du Travail et de la Main-d'oeuvre, coll. Études et recherches, 187 pp.
}

\section{Alain Vinet}

Volume 35, numéro 2, 1980

URI : https://id.erudit.org/iderudit/029076ar

DOI : https://doi.org/10.7202/029076ar

Aller au sommaire du numéro

Éditeur(s)

Département des relations industrielles de l'Université Laval

ISSN

0034-379X (imprimé)

1703-8138 (numérique)

Découvrir la revue

Citer ce compte rendu

Vinet, A. (1980). Compte rendu de [Le mouvement de restructuration du travail, par Paul Deschênes, Québec, ministère du Travail et de la Main-d'oeuvre, coll. Études et recherches, 187 pp.] Relations industrielles / Industrial Relations, 35(2), 348-349. https://doi.org/10.7202/029076ar

Tous droits réservés (C Département des relations industrielles de l'Université Laval, 1980
Ce document est protégé par la loi sur le droit d'auteur. L’utilisation des services d’Érudit (y compris la reproduction) est assujettie à sa politique d'utilisation que vous pouvez consulter en ligne.

https://apropos.erudit.org/fr/usagers/politique-dutilisation/ 
saire d'éviter tout risque de confusion qui ajouterait encore à la complexité des phénomènes observés. L'un des mérites de cette recherche est d'avoir pris en compte cette complexité en une démarche extensive qui élargit à mesure ses hypothèses et s'enrichit de nouvelles problématiques.

Le constat d'hétérogénéité auquel elle aboutit n'est pas fait pour surprendre. La question qui se profile est celle des deux dimensions selon lesquelles un groupe vit son identité socio-professionnelle. C'est par les transactions entre les déterminants sécrétés par l'entreprise et ceux que fait peser l'environnement que l'on pourrait approfondir l'interrogation. Cela reviendrait à étudier non plus seulement un groupe, sachant que les phénomènes que l'on saisit ainsi ont un caractère quelque peu abstrait, mais aussi des situations constituant des réalités sociales limitées mais complètes.

En d'autres termes, c'est en confrontant de multiples coupes effectuées sur le terrain que l'on pourra compléter les problématiques et éprouver la validité des hypothèses. Les cadres, enjeu politique? Il est significatif que ce soit là encore une question qui se pose nécessairement de l'extérieur. On pourrait la reformuler dans un sens qui la resitue: les cadres, objets ou acteurs du politique?

\section{Christian BUISSON}

Institut d'administration des entreprises Paris

Le mouvement de restructuration du travail, par Paul Dechêne, Québec, Ministère du Travail et de la Main-d'oeuvre, coll. études et recherches, $187 \mathrm{pp}$.

Cette publication du ministère du Travail et de la Main-d'oeuvre constitue un outil pédagogique des plus intéressants pour des personnes désireuses de se familiariser avec les problèmes liés à l'organisation du travail et à son évolution. L'ouvrage est divisé en deux parties: une analyse thématique de la littérature existante et une bibliographie sélective remarquablement commentée.
L'auteur établit tout d'abord son propre bilan de la littérature consacrée à la réorganisation du travail. Constatant que l'ampleur de cette littérature témoigne de la vigueur du débat soulevé par les nouvelles formes d'organisation du travail, il ne se limite pas à en établir un "bilan-portrait». Son intention est de formuler une synthèse critique de cette littérature «tant de cerner les pièges et les limites des réformes apportées à l'organisation du travail, que d'évaluer les conséquences de ces expériences pour les travailleurs, pour l'action syndicale». Il y réussit assez bien quoiqu'il ne lui soit pas possible, en si peu de pages, d'approfondir les problèmes qu'il soulève à juste titre. Le lecteur demeure donc sur sa faim.

Dans la première partie de l'ouvrage, l'auteur insiste sur la crise actuelle de l'organisation du travail, particulièrement sur les signes d'une résistance accrue des travailleurs à l'OST. Il décrit les approches les plus populaires en matière de restauration du travail enrichissement des tâches, approche sociotechnique et groupes autonomes - et s'attarde sur l'ampleur et l'impact de ces expériences. Il tente d'en évaluer les résultats pour l'entreprise et pour le travailleur et s'intéresse aux conséquences de ces tentatives sur l'action syndicale. De façon générale, l'analyse de Dechêne est critique mais nuancée. À l'exemple d'Annie Borzeix, il désire "échapper au double piège d'un réformisme à la fois naîf et optimiste, et d'un idéalisme révolutionnaire qui se contente de renvoyer à plus tard la résolution du problème soulevé». Cette attitude de prudence est de mise dans une revue de littérature, surtout si elle est appelée à jouer un rôle d'initiation au sujet dans divers milieux.

On peut difficilement reprocher à ce genre de publication de demeurer trop général. Le format et l'intention l'y obligent. Par contre, le lecteur désireux d'approfondir quelques uns des thèmes mentionnés précédemment, trouvera dans la seconde partie de l'ouvrage une bibliographie sélective soigneusement commentée par l'auteur. De Taylor et Friedmann à Trist et Davis, en passant par les théories sur la satisfaction au travail et leur 
application dans des expériences américaines et françaises, Dechêne dépouille une cinquantaine d'ouvrages et offre pour chacun un résumé succint mais substantiel. Ces résumés et commentaires sont présentés de manière à ce que le lecteur puisse rapidement se faire une opinion sur l'ouvrage en question. Cette seconde partie du travail de Dechêne présente une valeur pédagogique certaine.

En résumé, cette publication peut être conseillée à une personne non familière avec le sujet et désireuse d'obtenir une vue d'ensemble synthétique et critique de l'évolution des modes d'organisation du travail. Elle peut constituer une source de documentation féconde pour des étudiants. On n'y trouvera cependant pas de points de vue nouveaux ou de développement originaux.

\section{Alain VINET}

Université Laval

\section{Administration du travail et conflits collec- tifs, par Jean-Paul Bachy, Sceaux, CRESST, 1979, 164 pp.}

La littérature française des relations industrielles, passablement déficitaire sur le thème de l'intervention des pouvoirs publics dans les conflits collectifs du travail, s'enrichit d'une étude d'autant plus utile qu'en dépassant les frontières héxagonales, elle se place sur un terrain international et comparé.

En effet, après un chapitre sur «l'État et les conflits" où ne sont pas rares les références à l'Amérique du Nord, Bachy nous offre, dans un second chapitre, quatre cas étrangers: la Belgique, le Danemark, l'Allemagne fédérale et la Grande Bretagne, qui représentent, au-delà du sujet stricto sensu autant d'occasions d'introduction aux systèmes nationaux de relations du travail des pays respectifs, dont les singularités (et, aussi, implicitement, les convergences) sont bien mis en évidence.

Le troisième chapitre, le plus important en volume, est, naturellement, consacré à la
France. La disproportion entre le poids de la loi et celui de la négociation collective est, dès le début, abordé, et ceci prend toute son importance en ce début de décennie, quand de nouveaux équilibres se font jour dans le jeu des acteurs sociaux et que les employeurs réclament - et oeuvrent pour - des négociations «individualisées» au niveau de l'entreprise, au détriment de dispositions légales et contractuelles au plan des branches d'activité.

Les mécanismes de conciliation (conventionnelle et réglementaire), d'arbitrage et de médiation sont expliqués et évalués, et leur faible efficacité relevée. Le rôle actif - et insubstituable - de l'inspection du travail et l'élargissement de ces compétences sont ensuite mis en relief, de même que les obstacles que l'auteur a décelés au cours de son enquête, tels que le faible nombre de ses membres, qu'il voudrait voir dotés d'une autonomie supérieure, et - de là - les freins apportés parfois par l'autorité supérieure.

Bachy n'a pas manqué, dans sa recherche dont l'intérêt ne faiblit jamais, ni de demander l'avis des partenaires sociaux, ni poser le problème, il y a quelques années très débattu, de l'objectivité d'un corps fortement (et diversement) syndiqué, ni, in fine, de nous livrer quelques renseignements sur la sélection et la formation des inspecteurs.

Des considérations comparées clôturent ce document, rapport de recherche, pertinent, simple - je veux dire dépourvu de toute sophistication académique (mais, également, d'une table des matières...), écrit dans un langage clair et accessible, qui dit l'essentiel sans prétention d'exhaustivité. Un ouvrage nécessaire et sérieux qui s'ajoute à celui que l'auteur avait publié, il y a deux ans, sur Les jeunes et la société nouvelle (CRESST, 1977) et qui remplit, très méritoirement, un vide.

Des cinq pays qu'il a traités, il manque, toutefois, à mes yeux, le sixième. Ceci d'autant plus que l'Italie nous avait offert, il y a déjà plusieurs années, un remarquable ouvrage - de juriste, mais écrit dans une perspective de relations industrielles -, que je 